\title{
CIRUGÍA LAPAROSCÓPICA DE QUISTES ADRENALES: RESULTADOS DE UNA SERIE DE 18 PACIENTES*
}

\author{
Drs. Octavio A. Castillo C. ${ }^{\mathbf{1}, 2}$, Jorge López-Vallejo C. ${ }^{\mathbf{1}}$, \\ Vincenzo Borgna C. ${ }^{2}$, Ricardo Yáñez P. ${ }^{1}$ \\ 1 Unidad de Urología, Clínica INDISA. \\ 2 Facultad de Medicina, Universidad Andrés Bello. \\ Santiago, Chile.
}

\section{Laparoscopic surgery of adrenal cysts: results of a series of 18 patients}

Introduction: Adrenal cysts have an incidence of $0.064 \%$ to $0.18 \%$ at autopsy. Aim: To show our experience in 18 patients with an adrenal cyst operated by laparoscopy. Material and Methods: The series consisted of 18 patients operated, in a series of 344 laparoscopic adrenalectomy performed between 1993 and 2011. The age range was 20 to 61 years with an average of 38 years, 11 women and 7 men. Results: Thirteen cysts were left and 5 were right sided. The lesion size varied between 4.5 and $12.5 \mathrm{~cm}$, with an average of 9.2 $\mathrm{cm}$. Eight patients complained of abdominal discomfort and in the remaining 12 patients there were no signs or symptoms of clinical suspicion. The surgery performed was a laparoscopic decortication-marsupialization in 11 patients, laparoscopic adrenalectomy in 8 cases and partial adrenalectomy in 1 case. Mean operative time was 83.1 minutes (30-180 minutes), and mean hospital stay was 36 hours. Intraoperative complications occurred in 2 patients: 1 diaphragm injury and one renal vein injury, repaired in the same surgery. Postoperative complications occurred in 2 patients, both Clavien 3a, treated by conservative management. Conclusion: Small and non-functional lesions require observation. Partial or total laparoscopic adrenalectomy is indicated in large lesions or in functional and suspected malignant lesions.

Key words: Adrenal cyst, adrenal neoplasm, adrenal surgery, laparoscopy.

\section{Resumen}

Introducción: Los quistes suprarrenales tienen una incidencia de $0,064 \%$ a $0,18 \%$ en autopsias. El objetivo de este trabajo es mostrar nuestra experiencia en 18 pacientes portadores de un quiste adrenal operados por vía laparoscópica. Material y Métodos: La serie está constituida por 18 pacientes, de una serie de 344 adrenalectomías laparoscópicas realizadas entre los años 1993 y 2011. El rango de edad fue de 20 a 61 años, con un promedio de 38 años, 11 mujeres y 7 hombres. Resultados: Trece quistes eran del lado izquierdo y 5 del derecho. El tamaño de la lesión varió entre 4,5 y 12,5 cm, con un promedio de 9,2 cm. En 8 pacientes hubo molestias abdominales vagas y en los restantes 12 pacientes no hubo signos ni síntomas de sospecha clínica.

\footnotetext{
Los autores no refieren conflictos de interés.

Correspondencia: Dr. Octavio A. Castillo C. octavio.castillo@indisa.cl
}

*Recibido el 23 de octubre de 2013 y aceptado el 22 de noviembre de 2013 
La cirugía realizada fue una decorticación-marsupialización laparoscópica en 11 pacientes, adrenalectomía laparoscópica en bloque en 8 casos y adrenalectomía parcial en 1 caso. El tiempo operatorio promedio fue de 83,1 min (30-180 min), y el tiempo medio de hospitalización fue de 36 h. Complicaciones intraoperatorias ocurrieron en 2 pacientes: 1 lesión de diafragma y 1 lesión de vena renal, reparadas en el mismo acto quirúrgico. Complicaciones postoperatorias ocurrieron en 2 pacientes, ambas Clavien $3 \mathrm{a}$ y de manejo conservador. Conclusión: Las lesiones pequeñas y no funcionantes requieren observación. La adrenalectomía laparoscópica parcial o total está indicada en lesiones de gran tamaño, con importante compromiso de la glándula o ante la sospecha de lesión funcionante o malignidad.

Palabras clave: Quiste adrenal, tumor adrenal, cirugía adrenal, laparoscopia.

\section{Introducción}

El amplio uso de las técnicas diagnósticas por imágenes, ha aumentado en forma importante el diagnóstico de masas suprarrenales incidentales o "incidentalomas". Entre ellas, los quistes suprarrenales son de infrecuente hallazgo, con una incidencia en autopsias de $0,064 \%$ a $0,18 \%{ }^{1}$. Aunque la mayoría de los quistes adrenales son no funcionantes y asintomáticos, pueden alcanzar tamaños suficientes como para producir síntomas abdominales inespecíficos, dolor lumbar o hipertensión. Causas de indicación quirúrgica han sido el tamaño del quiste (mayores de $6 \mathrm{~cm}$ ), quistes sintomáticos, quistes hormonalmente funcionantes, o aquellos sospechosos de malignidad ${ }^{2}$.

Debido a su baja incidencia, la mayoría de las publicaciones están compuestas de un escaso número de $\operatorname{casos}^{3-6}$, lo cual hace difícil sacar conclusiones en cuanto a su manejo, y especialmente a la indicación de la cirugía, ya sea abierta o laparoscópica.

El objetivo de este trabajo es mostrar nuestra experiencia en 18 pacientes diagnosticados de un quiste adrenal y operados por vía laparoscópica.

\section{Material y Método}

La serie está constituida por 18 pacientes portadores de un quiste adrenal operados por vía laparoscópica, de una serie personal de 344 adrenalectomías laparoscópicas realizadas entre los años 1993 y 2011. Sólo se incluyeron en esta serie los quistes adrenales adecuadamente caracterizados en el estudio por imágenes, excluyendo aquellas lesiones tumorales quísticas de diagnóstico incierto.

El rango de edad fue de 20 a 61 años, con un promedio de 38 años, 11 mujeres y 7 hombres. Los datos fueron recolectados en forma prospectiva y analizados retrospectivamente.

En todos los casos se realizó estudio endocrinológico completo, tal cual lo descrito para estudio de una incidentaloma suprarrenal ${ }^{7}$.

La técnica quirúrgica ha sido descrita previamente, utilizando una técnica de 3 trocares a izquierda y
4 trocares a derecha, todos por vía transperitoneal ${ }^{8}$.

Las complicaciones intraoperatorias fueron clasificadas según Satava ${ }^{9}$ y las post-operatorias fueron definidas de acuerdo a los criterios de la clasificación modificada de Clavien ${ }^{10}$.

\section{Resultados}

De acuerdo a la clasificación de la American Society of Anesthesiology (ASA), 3 pacientes eran ASA 2, dos por hipertensión y uno por obesidad. El resto de los pacientes fue ASA 1. El índice de masa corporal promedio fue de $23,2 \mathrm{~kg} / \mathrm{m}^{2}$, con un rango de 23 a $35 \mathrm{~kg} / \mathrm{m}^{2}$. Trece quistes eran del lado izquierdo y 5 del derecho. El tamaño de la lesión varió entre 4,5 y $12,5 \mathrm{~cm}$, con un promedio de $9,2 \mathrm{~cm}$.

En 8 pacientes hubo molestias abdominales vagas o malestar en el flanco, que condujeron al estudio radiológico. En los restantes 12 pacientes no hubo signos ni síntomas de sospecha clínica.

La cirugía realizada fue una decorticaciónmarsupialización laparoscópica en 11 pacientes (con preservación de la glándula suprarrenal), adrenalectomía laparoscópica en bloque en 8 casos, determinada fundamentalmente por el tamaño de la lesión o la imposibilidad de identificar la glándula suprarrenal durante el acto quirúrgico, y adrenalectomía parcial en 1 caso.

El tiempo operatorio promedio fue de $83,1 \mathrm{~min}$ (rango de 30-180 min), y el tiempo medio de hospitalización fue de $36 \mathrm{~h}$.

Complicaciones intraoperatorias ocurrieron en 2 pacientes: 1 lesión de diafragma y 1 lesión de vena renal (Satava II), ambas reparadas laparoscópicamente en el intraoperatorio. Complicaciones postoperatorias ocurrieron en 2 pacientes: 1 hematoma retroperitoneal (Clavien 3a) manejado conservadoramente y sin necesidad de transfusión, y una hernia incisional pequeña en 1 puerto (Clavien 1), asintomática, que no ha requerido de cirugía. No hubo conversión ni mortalidad en la serie.

El estudio histopatológico demostró predominancia de Quiste endotelial (50\%), seguido por Seudoquiste hemorrágico $(44,4 \%)$ (Tabla 1$)$. La incidencia 


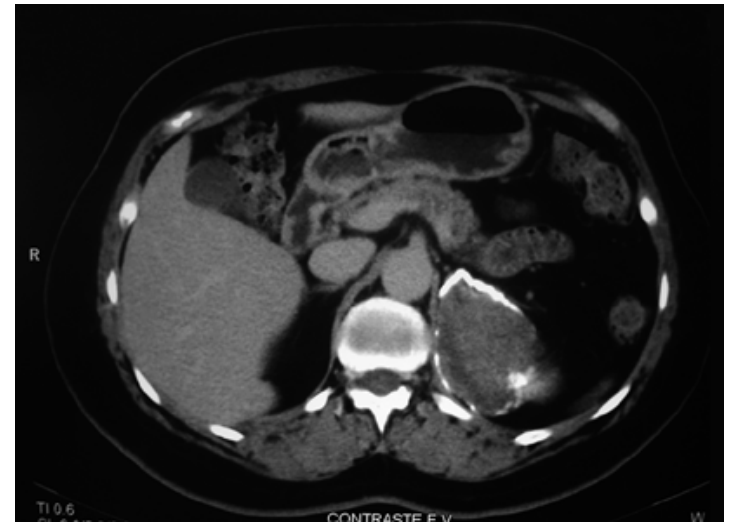

Figura 1. Tomografía computada que muestra una lesión sólido-quística con necrosis e inflamación (Seudoquiste hemorrágico calcificado).

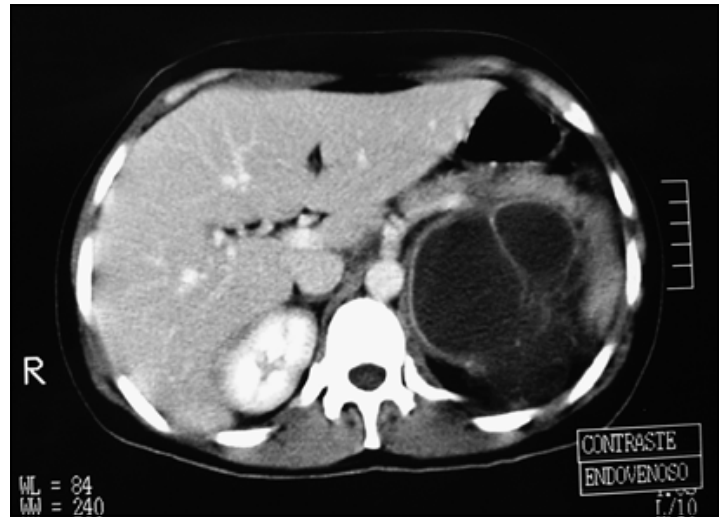

Figura 3. Tomografía computada de un linfangioma quístico multilocular.

Tabla 1. Características histopatológicas de la serie

\begin{tabular}{|ll|}
\hline Quiste endotelial-linfático & 9 casos \\
Seudoquiste hemorrágico & 7 casos \\
Linfangioma quístico multilocular & 1 caso \\
\hline
\end{tabular}

global de quistes adrenales en nuestra serie de adrenalectomías laparoscópicas fue de 5,2\%.

Las Figuras ilustran 3 tipos de lesiones quísticas suprarrenales de nuestra serie.

\section{Discusión}

El diagnóstico por imágenes de uso masivo ha determinado un aumento en su diagnóstico, con más de 1.000 casos comunicados desde el año 1995. La

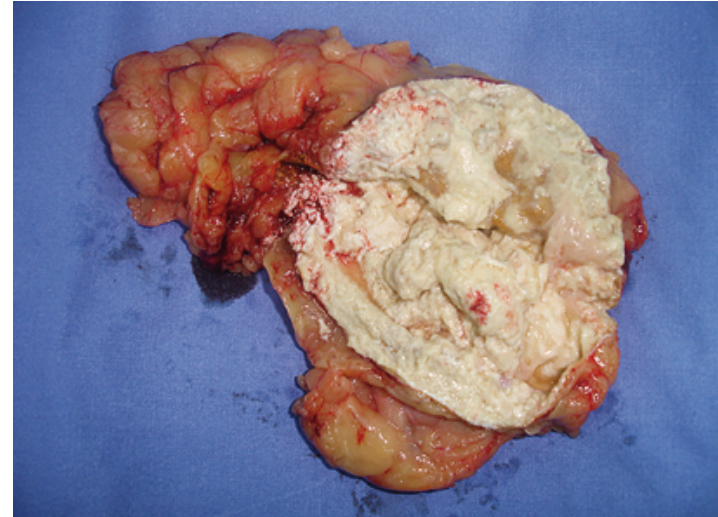

Figura 2. Visión macroscópica de seudoquiste hemorrágico calcificado de la Figura 1.

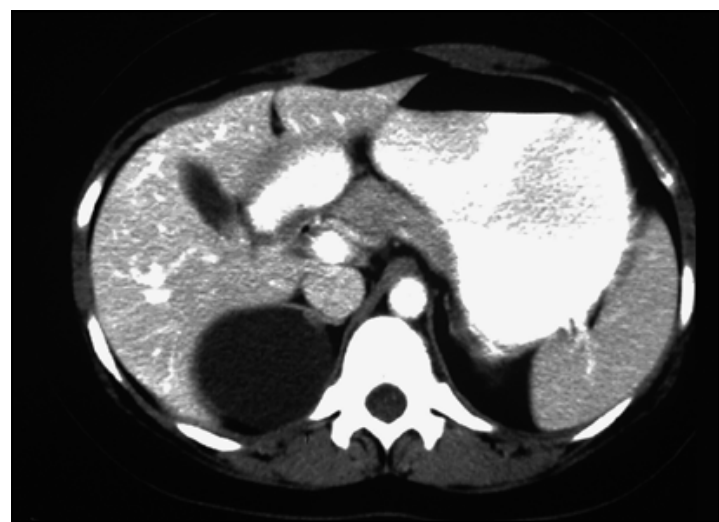

Figura 4. Tomografía computada de un quiste endotelial.

limitación más importante de las publicaciones sobre quistes adrenales, es que la gran mayoría sólo se refiere a casos clínicos, habiendo pocas series publicadas con un número de pacientes que permita sacar conclusiones valederas respecto a su tratamiento.

En el año 2005, publicamos una serie inicial de 8 pacientes portadores de quistes suprarrenales operados por vía laparoscópica ${ }^{4}$. El examen histológico demostró seudoquiste hemorrágico en 5 pacientes y quiste endotelial en 3.

El espectro histopatológico puede variar desde seudoquistes a lesiones malignas. En algunas series el quiste más frecuente es el endotelial (45\%), seguido de seudoquiste hemorrágico $(39 \%)$, quiste epitelial $(9 \%)$ y parasitarios $(7 \%)$. Sin embargo, otras series muestran al seudoquiste como el más frecuente $e^{4,5,11}$.

En la revisión de la literatura nos parece que la mejor clasificación propuesta es la de Davenport et 
Tabla 2. Clasificación patológica de los quistes (según Davenport en $\mathbf{a l}^{12}$ )

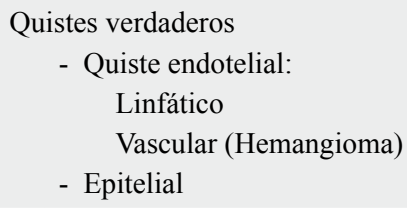

Seudoquistes

- Hemorrágico

- Neoplásico

- Parasitario

$\mathrm{al}^{12}$, basada en aquella de Hodges y Ellis ${ }^{13}$, quien propone una diferenciación clara entre quistes verdaderos y seudoquistes (Tabla 2).

Los quistes verdaderos, a diferencia de los seudoquistes, presentan una capa endotelial o epitelial interna. La mayoría de los quistes endoteliales son de origen linfático. Los quistes endoteliales verdaderos (hemangiomas) son infrecuentes. Los quistes de origen epitelial representan con probabilidad adenomas quísticos. Los seudoquistes no tienen cubierta celular. El tipo hemorrágico (más frecuente) tiene una pared fibrosa densa, que puede estar calcificada e incluso osificada. El contenido es generalmente hemático. A menudo se encuentra tejido adrenal cortical en su pared como islotes aplanados o pequeñas masas. Se estima que se originan de la encapsulación y organización de un hematoma en una glándula adrenal normal o anormal secundaria a trauma, sepsis, hipoxia o tumores.

La degeneración quística de un tumor maligno, primario o metastásico, y los quistes parasitarios son entidades que se deben clasificar entre los seudoquistes (según Hodges y Ellis) ${ }^{13}$.

El diagnóstico diferencial generalmente ocurre entre los quistes endoteliales linfáticos y los seudoquistes hemorrágicos, que representan el mayor número entre los quiste adrenales (en nuestra serie el $50 \%$ y el $44 \%$ respectivamente).

No se han descrito criterios radiológicos específicos para quistes adrenales. Se ha sugerido que una razonable forma de evaluar su potencial maligno podría estar basado en la clasificación de Bosniak de las masas quísticas renales ${ }^{14}$. Khoda y cols usaron la clasificación de Bosniak para estratificar sus pacientes y pudieron seleccionar en forma segura los pacientes susceptibles de manejo conservador. $\mathrm{Su}$ limitación es que no existen comunicaciones respecto de la sensibilidad y especificidad en la diferenciación de benignidad o malignidad ${ }^{15}$. Rozenblit et $\mathrm{al}^{16}$, propone una clasificación de las ma- sas adrenales quísticas no funcionantes en 4 tipos, basándose en criterios radiológicos: Se consideran quistes no complicados aquellos menores de 5-6 cm, homogéneos y con pared menor de $3 \mathrm{~mm}$; en esta variedad se recomienda la vigilancia periódica para detectar cambios en la naturaleza o en el tamaño. Como quistes complicados se clasifican aquellos con altos valores de atenuación o no homogéneos, con pared mayor de $5 \mathrm{~mm}$ de espesor o con gruesas calcificaciones centrales o periféricas: se recomienda su extirpación quirúrgica. Los quistes mayores de 5-6 cm con valores de atenuación mayores que el agua o con pared entre 3 y $5 \mathrm{~mm}$ se consideran indeterminados. En esta variedad estaría indicada una punción de la lesión previa a la decisión terapéutica.

Ansari et $a^{17}$, propone una clasificación clínica en función de los síntomas que producen. Ante los asintomáticos propone vigilancia radiológica con TC cada 6 meses, y cirugía sólo si produce aumento de tamaño en el seguimiento; ante los sintomáticos y/o funcionantes sugiere cirugía de primera intención.

El amplio uso del ultrasonido y la tomografía computada a resultado en un aumento del diagnóstico incidental de los quistes adrenales. La sensibilidad de la tomografía computada se ha reportado entre $57 \%$ a $80 \%$, 18 . En nuestra serie su sensibilidad fue de $95 \%$, por 1 caso diagnosticado como quiste complicado de polo superior renal izquierdo, asociado a múltiples quistes renales simples. El problema más importante es lograr la diferenciación entre lesiones benignas y malignas, con series que reportan una sensibilidad del $100 \%$ en la exclusión de malignidad $^{19}$. La mayoría de los carcinomas adrenales se ven como tumores sólidos con captación del medio de contraste en estudios radiológicos, diámetro mayor de $5 \mathrm{~cm}$, y algunas veces funcionante ${ }^{20}$.

Aunque la mayoría de los quistes suprarrenales son asintomáticos y no funcionantes, pueden aumentar de tamaño y causar síntomas inespecíficos como malestar o dolor abdominal o lumbar, o hipertensión ${ }^{21}$.

En general, independiente de su tamaño, se debe realizar estudio endocrino completo, el cual debiera incluir catecolaminas urinarias de $24 \mathrm{~h}$, metanefrinas, ácido vanilmandélico, 17-hidroxiesteroides, aldosterona plasmática, renina y actividad de cortisol $^{7}$.

Quistes pequeños, asintomáticos o no funcionantes, pueden ser controlados clínica, radiológica y funcionalmente a intervalos de un año. Ante la imposibilidad de descartar una lesión maligna, la cirugía está plenamente indicada. Esta puede ir desde la cirugía abierta ${ }^{12,16}$ a la cirugía laparoscópi$\mathrm{ca}^{4,5,21}$. La experiencia ha demostrado que la cirugía laparoscópica permite una adecuada resección de la mayoría de las lesiones suprarrenales, aún en casos de tumor maligno primario o secundario ${ }^{22}$. 


\section{Conclusión}

Aunque infrecuentes, los quistes adrenales plantean un dilema diagnóstico y terapéutico. En lesiones pequeñas y no funcionantes, la elección es la observación periódica clínica y radiológica. La cirugía laparoscópica de los quistes adrenales es segura y posible. El tratamiento preferido debiera ser la decorticación o marsupialización en quistes asintomáticos o seudoquistes. La adrenalectomía laparoscópica parcial o total está indicada en lesiones de gran tamaño, con importante compromiso de la glándula o ante la sospecha de lesión funcionante o malignidad.

\section{Referencias}

1. Walsh H. Adrenal cysts. Am J Pathol. 1951;27:758.

2. Bellantone R, Ferrante A, Raffaelli M, Boscherini M, Lombardi CP, Crucitti F. Adrenal cystic lesions: Report of 12 surgically treated cases and review of the literature. J Endocrinol Invest. 1998;21:109-14.

3. Neri LM, Nance FC. Management of adrenal cysts. Am Surg. 1999;65:151-63.

4. Castillo OA, Litvak JP, Kerkebe M, Urena RD. Laparoscopic management of symptomatic and large adrenal cysts. J Urol. 2005;173:915-17.

5. Guazzoni G, Montorsi F, Rigatti P, Lanzi R, Pontiroli AE, Sivestre $P$, et al. Laparoscopic unroofing of adrenal cysts. Eur Urol. 1997;31:499-502.

6. Koksoy FN, Yucel O, Celik A, Igdem AA. Laparoscopic management of a giant adrenal cyst: Case report. Surg Laparosc Endosc Percutan Tech. 2001;11:379-81.

7. Grumbach MM, Biller BM, Braunstein GD, Campbell KK, Carney JA, Godley PA, et al. Management of the clinically inapparent adrenal mass ("incidentaloma"). Ann Intern Med. 2003;138:424-9.

8. Castillo O, Cortés O, Kerkebe M, Pinto I, Arellano L, Russo M. Adrenalectomía laparoscópica: lecciones aprendidas en 110 procedimientos. Rev Chil Cir. 2006;58:175-80.

9. Satava RM. Identification and reduction of surgical error using simulation. Minim Invasive Ther Allied Technol.
2005; 14:257-61.

10. Dindo D, Demartines N, Clavien PA. Classification of surgical complications: a new proposal with evaluation in a cohort of 6336 patients and results of a survey. Ann Surg. 2004;240:205-13.

11. Lal TG, Kaulback KR, Bombonati A, Palazzo JP, Jeffrey RB, Weigel RJ. Surgical management of adrenal cysts. Am Surg. 2003;69:812-4.

12. Davenport M, Pollard K, Smith SEW, MacMahon MT: Adrenal cysts- report, review and classification. Postgraduate Medical Journal 1988;64:71-3.

13. Hodges FV, Ellis FR. Cystic lesions of the adrenal gland Arch Pathol. 1958;66:53-8.

14. Bosniak MA. The current radiological approach to renal cysts. Radiology 1986;158:1-10.

15. Khoda J, Hertzanu Y, Sebbag G, Lantsberg L, Barky Y. Adrenal cysts: Diagnosis and therapeutic approach. Int Surg. 1993;78:239-42.

16. Rozenblit A, Morehouse HT, Amis ES Jr. Cystic adrenal lesions: CT features. Radiology 1996;201:541-48.

17. Ansari MS, Singh I, Hemal AK. Cost-reductive retroperitoneal excision of large adrenal pseudocyst: A case report and review of the literature. Int Urol and Nephrol. 2001;33:307-10.

18. Tiberio GA, Bonardelli S, Baiocchi GL, Grazioli L, Rizzoni D, Coniglio A, et al. Cystic type adrenal mass. Clinical-radiologic contribution to 7 cases treated with surgery. Chir Ital. 2003;55:681-6.

19. El-Hefnawy AS, El Garba M, Osman Y, Eraky I, El Mekresh M, Ibrahim EL-H Surgical management of adrenal cysts: single-institution experience. BJU Int. 2009; 104:847-50.

20. Liao CH, Chueh SC, Lai MK, Hsiao PJ, Chen J. Laparoscopic adrenalectomy for potentially malignant adrenal tumors greater than 5 centimeters. J Clin Endocrinol Metab. 2006;91:3080-83.

21. Koksoy, F N, Yucel O, Celik A, Igdem AA. Laparoscopic management of a giant adrenal cyst: case report. Surg Laparosc Endosc Percutan Tech. 2001;11:379-81.

22. Castillo OA, Vitagliano G, Kerkebe M, Parma P, Pinto I, Díaz M. Laparoscopic adrenalectomy for suspected metastasis of adrenal gland: our experience. Urology 2007;69:637-41. 